The $3^{\text {rd }}$ Conf. of SSFOP "Recent Techniques in Ornamental Plants Scope", Cairo, Egypt, 26/2/2017

Scientific J. Flowers \& Ornamental Plants

www.ssfop.com/journal

ISSN: 2356-7864

\title{
EFFECT OF PLANT DENSITY AND SOME VITAMINS, AS WELL AS, ACTIVE YEAST ON SWEET BASIL (OCIMUM BASILICUM) PLANT A- VEGETATIVE GROWTH CHARACTERS
}

\author{
M.A.H. Abdou, A.A. El-Sayed, R.A. Taha and Z.S.A. Marzok \\ Hort. Dept., Fac. Agric., Minia Univ., Egypt.
}

ABSTRACT: This investigation was conducted to investigate the effect of plant density $\left(145,105,80\right.$ and 65 plants $\left./ 14.5 \mathrm{~m}^{2}\right)$ and some vitamins (vit. $B_{1}$, vit. $C$, vit. $E$ ) and active yeast on vegetative growth traits of Ocimum basilicum plants.

Results showed that decreasing plant density led to increase number of branches/plant/cut, leaf area/plant/cut, herb fresh and dry weights/plant/cut and yield of herb (fresh and dry) per plant per season, while, plant height was decreased. Moreover, the herb fresh and dry weights/fed were increased with the highest density (145 plants $/ 14.5 \mathrm{~m}^{2}$ ).

Scientific J. Flowers \& Ornamental Plants,

All used treatments of vitamins and active yeast significantly increased all previous characters of vegetative growth. Active yeast 4(1):83-100 (2017). followed by collection of vitamins (vit. $\mathrm{B}_{1}+$ vit. $\mathrm{C}+$ vit. E) resulted in the highest values in this concern.

Received:

10/1/2017

Accepted:

31/1/2017

All vegetative growth characters per plant were the most effective treatments on the lowest plant density $\times$ active yeast or vit. $B_{1}+$ vit. $C$ + vit. $E$ while, the highest values per fed were obtained with higher density in combination with active yeast or vit. $\mathrm{B}_{1}+$ vit. $\mathrm{C}+$ vit. $\mathrm{E}$.

Key words: Ocimum basilicum, plant density, vitamins, active yeast, vegetative growth.

\section{INTRODUCTION}

Ocimum basilicum, L. is a member of Lamiaceae family. This species originated in Egypt and East Mediterranean. Basil has a long history of use as food flavors, perfumes and medicinal values.

In suitable plant density, plants are completely used under environmental conditions (water, air, light and soil) and inter or intra specific competition is minimum (Sadeghi et al., 2009; Chegini et al., 2012; Abbas, 2014; Bekhradi et al., 2014 and Atghaei et al., 2015) on basil plants.

Active yeast is a natural safety biofertilizer causes various promoted effects on basil plants (Salman, 2006, El-Keasy et al., 2011, Abd El-Salam, 2014, El-Naggar et al., 2015 and Nassar et al., 2015).

Many authors concluded that, some vitamins as antioxidants have positive effect on plants such as, Abd El-Salam, 2014 on basil plants, Shehata (2013) on guar and Botros (2013) on caraway and Helmy (2016) on cumin plants. This study aimed to investigate the effect of plant density and some natural substances to achieve maximum herb yields of basil under the conditions of Minia Governorate.

\section{MATERIALS AND METHODS}

The experiment was carried out during the two seasons of 2014 and 2015 at the floriculture Nursery at the Experimental 
Farm of Faculty of Agriculture, Minia university to investigate the effect of plant density and active yeast, as well as, some vitamins and their interaction on the vegetative growth and total herb yield of Ocimum basilicum, L. plants.

The experiment was arranged in a complete randomized block design in a split plot design with three replicates. The main plots (A) included four plant densities, while six treatments of active yeast and vitamins, as well as, control occupied the sub plots (B). Therefore, the interaction treatments $(\mathrm{A} \times \mathrm{B})$ were 24 treatments. The experimental unit (plot) was $7.25 \times 2.0 \mathrm{~m}$ and contained 5 rows. The seedlings at the stage of 4-5 leaves and 11-12 cm height were transplanted (March $1^{\text {st }}$, in both seasons) and cultivated in hills, therefore, each plot contained 145, 105, 80 and 65 plants $/ 14.5 \mathrm{~m}^{2}$. The physical and chemical analysis of the used soil in both seasons are determined according to Jackson (1973) and shown in Table (a).

\section{Treatments:}

\section{Main plots (A):}

The main plots (A) included the following four plant densities:

1. 145 plants $/ 14.5 \mathrm{~m}^{2}=40000$ plants/fed (25 cm distance).

2. 105 plants $/ 14.5 \mathrm{~m}^{2}=28966$ plants/fed (35 cm distance).

3. 80 plants $/ 14.5 \mathrm{~m}^{2}=22069$ plants/fed (45 cm distance).
4. 65 plants $/ 14.5 \mathrm{~m}^{2}=17931$ plants/fed (55 cm distance).

\section{Sub plots (B):}

The sub plots (B) were devoted to six treatments as follows:

1. Control (spray with tap water).

2. Thiamine, vit. $B_{1}$ at $50 \mathrm{ppm}$.

3. Ascorbic acid, vit. $\mathrm{C}$ at $50 \mathrm{ppm}$.

4. Alpha tocopherol, vit. E at $10 \mathrm{ppm}$.

5. Vit. $\mathrm{B}_{1}+$ vit. $\mathrm{C}+$ vit. $\mathrm{E}$ at 50,50 and $10 \mathrm{ppm}$, respectively.

6. Active dry yeast (ADY) at $5 \mathrm{~g} / \mathrm{l}$.

Each of suspension of yeast and vitamins were applied by hand sprayer, 3 times. The first one was after 6 weeks from transplanting date (April 14 ${ }^{\text {th }}$ ), the second one was added after two weeks from the first cut (May 28 $8^{\text {th }}$ ) and the third one was added after two week from the second cut (middle of July). The plants were sprayed till run off. All agricultural practices were performed as usual, in the region for the production of sweet basil plants.

\section{Harvesting times:}

During each experimental season, the plants were harvested three times at approximately $50 \%$ flowering. In each harvest, the plants were cut leaving about 10 $\mathrm{cm}$ above the soil surface. The first cut was done on $14^{\text {th }}$ of May, the second cut was done on $1^{\text {st }}$ of July. Meanwhile, the third cut was done on $28^{\text {th }}$ of August in the two growing seasons.

Table a. Physical and chemical properties of the used soil.

\begin{tabular}{|c|c|c|c|}
\hline Soil Character & Value & Soil Character & Value \\
\hline Sand \% & 29.00 & Available P \% & 14.98 \\
\hline Silt \% & 30.00 & Exch. $K^{+}$(mg/100 g soil) & 2.16 \\
\hline Clay \% & 41.00 & Exch. $\mathrm{Ca}^{++}(\mathrm{mg} / 100$ g soil $)$ & 31.55 \\
\hline Texture grade & Clay loam & Exch. $\mathrm{Na}^{+}(\mathrm{mg} / 100$ g soil $)$ & 2.39 \\
\hline Organic matter \% & 1.68 & \multirow{5}{*}{$\begin{array}{l}\text { DTPA } \\
\text { Ext. ppm }\end{array}$} & 7.55 \\
\hline $\mathrm{CaCO}_{3} \%$ & 2.08 & & 2.16 \\
\hline E.C. (mmhos/cm) & 7.85 & & 2.64 \\
\hline pH (1:2.5) & 1.04 & & 7.35 \\
\hline Total N \% & 0.09 & & \\
\hline
\end{tabular}




\section{Data recorded:}

\section{Vegetative growth parameters:}

The following data were recorded at the harvesting time of each cut:

- Plant height (cm).

- Number of branches/plant/cut.

- Leaf area $\left(\mathrm{cm}^{2}\right)$.

- Fresh weight of herb/plant/cut (g/plant).

- Dry weight of herb/plant/cut (g/plant).

In addition, the following data of vegetative growth characters were also calculated:

- Total fresh weight of herb (g/plant/season).

- Total dry weight of herb (g/plant/season).

- Yield fresh weight of herb (ton/fed/season).

- Yield dry weight of herb (ton/fed/season).

\section{Statistical analysis:}

The obtained data were tabulated and statistically analyzed according to MSTATC (1986) and the L.S.D. test at $5 \%$ was followed to compare between the means.

\section{RESULTS AND DISCUSSION}

\section{Vegetative growth traits:}

\section{Plant height (cm):}

The effect of plant density on the plant height had significant in the three cuts in the two experimental seasons. The tallest plants observed in 145 plants $/ 14.5 \mathrm{~m}^{2}$ followed by 105 plants $/ 14.5 \mathrm{~m}^{2}, 80$ plants $/ 14.5 \mathrm{~m}^{2}$ then 65 plants $/ 14.5 \mathrm{~m}^{2}$ which gave the shortest plants in the three cuts in the two seasons (Tables, 1 and 2). Plant height was increased with increasing plant density of Ocimum spp. (Balyan et al., 1987, Sadeghi et al., 2009,
Patel and Kushwaha, 2013 and Abbas, 2014).

Data presented in Tables (1 and 2) showed that all used treatments of vitamins and active yeast significantly increased plant height during the three cuts in both seasons comparing with untreated plants. Among five used treatments, active yeast gave taller plants than those obtained by any other treatments and control. In this respect, ElKeasy et al. (2011); Abd El-Salam (2014); El-Naggar et al. (2015) and Nassar et al. (2015) on basil plants found that active yeast treatment increased plant height.

The interaction between plant density and some vitamins, as well as, active yeast treatments was significant for plant height in the three cuts in both seasons (Tables, 1 and $2)$. The tallest plants were produced due to the highest density treatments (145 and 105 plants $/ 14.5 \mathrm{~m}^{2}$ ) which supplied with active yeast or vit. $\mathrm{B}_{1}+$ vit. $\mathrm{C}+$ vit. $\mathrm{E}$ (in some cuts).

\section{Number of branches, leaf area, herb fresh and dry weights/plant/cut:}

The results showed that, number of branches/plant/cut (Tables, 3 and 4), leaf area (Tables, 5 and 6), herb fresh and dry weights/cut (Tables, 7, 8, 9 and 10) were greatly affected with plant density. Such four characters were gradually increased upward by the gradual decrease in plant density with significant differences among density treatments in the three cuts in the two growing seasons. So, the highest values were obtained with lowest density (65 plants/14.5 $\mathrm{m}^{2}$ ). Similar results were obtained by Balyan et al. (1987) and Davis (1993) on sweet basil.

All used five treatments significantly increased number of branches, leaf area, fresh and dry weights per plant during the three cuts in both seasons comparing with control treatments (Tables, 3 to 10). Among such five treatments, active yeast treatment significantly increased such four characters comparing with other used treatments. 
M.A.H. Abdou et al.

Table 1. Effect of plant densities and some vitamins and yeast treatments on plant height (cm) of sweet basil (Ocimum basilicum, L.) plants during the first season.

\begin{tabular}{|c|c|c|c|c|c|}
\hline \multicolumn{6}{|c|}{$1^{\text {st }}$ Cut } \\
\hline \multirow{2}{*}{ Treatments } & \multicolumn{5}{|c|}{ Plant densities /14.5 $\mathrm{m}^{2}$ (A) } \\
\hline & 145 & 105 & 80 & 65 & Mean (B) \\
\hline Control & 68.67 & 64.08 & 63.40 & 54.75 & 62.73 \\
\hline Vit. $B_{1}$ & 70.33 & 66.42 & 63.10 & 62.67 & 65.63 \\
\hline Vit. C & 75.45 & 74.75 & 74.47 & 72.43 & 74.28 \\
\hline Vit. E & 72.89 & 70.33 & 67.60 & 66.38 & 69.30 \\
\hline Vit. $B_{1}+$ Vit. $C+$ Vit. $E$ & 78.89 & 77.75 & 73.53 & 71.38 & 75.39 \\
\hline Yeast & 86.22 & 84.92 & 76.80 & 76.10 & 81.01 \\
\hline Mean (A) & 75.41 & 73.04 & 69.82 & 67.29 & 71.39 \\
\hline L.S.D. at $5 \%$ & \multicolumn{2}{|c|}{ A : 1.30} & B : 1.58 & \multicolumn{2}{|r|}{$\mathrm{AB}: 3.15$} \\
\hline \multicolumn{6}{|c|}{$2^{\text {nd }}$ Cut } \\
\hline Control & 64.56 & 58.92 & 55.41 & 53.47 & 58.09 \\
\hline Vit. $B_{1}$ & 70.44 & 68.25 & 67.07 & 64.66 & 67.61 \\
\hline Vit. C & 76.11 & 73.17 & 70.53 & 69.81 & 72.41 \\
\hline Vit. E & 74.44 & 70.00 & 68.05 & 67.60 & 53.12 \\
\hline Vit. $B_{1}+$ Vit. C+Vit. E & 81.67 & 77.83 & 75.47 & 69.95 & 76.23 \\
\hline Yeast & 82.33 & 82.25 & 78.87 & 76.38 & 79.96 \\
\hline Mean (A) & 74.93 & 71.74 & 69.23 & 66.98 & 70.72 \\
\hline L.S.D. at $5 \%$ & \multicolumn{2}{|c|}{ A : 1.92} & B : 2.45 & \multicolumn{2}{|r|}{$\mathrm{AB}: 4.90$} \\
\hline \multicolumn{6}{|c|}{$3^{\text {rd }}$ Cut } \\
\hline Control & 62.22 & 55.33 & 53.53 & 48.32 & 54.85 \\
\hline Vit. $B_{1}$ & 62.33 & 61.00 & 60.67 & 46.06 & 57.52 \\
\hline Vit. C & 63.14 & 63.11 & 62.17 & 60.92 & 62.34 \\
\hline Vit. E & 62.67 & 62.00 & 59.25 & 56.86 & 60.20 \\
\hline Vit. $B_{1}+$ Vit. $C+$ Vit. $E$ & 75.33 & 73.92 & 72.33 & 72.33 & 73.48 \\
\hline Yeast & 78.22 & 77.07 & 76.75 & 75.62 & 76.92 \\
\hline Mean (A) & 67.32 & 65.41 & 64.12 & 60.02 & 64.21 \\
\hline L.S.D. at $5 \%$ & \multicolumn{2}{|c|}{ A : 3.53} & B : 2.27 & \multicolumn{2}{|r|}{$\mathrm{AB}: 4.55$} \\
\hline
\end{tabular}


Table 2. Effect of plant densities and some vitamins and yeast treatments on plant height (cm) of sweet basil (Ocimum basilicum, L.) plants during the second season.

\begin{tabular}{|c|c|c|c|c|c|}
\hline \multicolumn{6}{|c|}{$1^{\text {st }}$ Cut } \\
\hline \multirow{2}{*}{ Treatments } & \multicolumn{5}{|c|}{ Plant densities /14.5 $\mathrm{m}^{2}$ (A) } \\
\hline & 145 & 105 & 80 & 65 & Mean (B) \\
\hline Control & 62.22 & 59.83 & 58.76 & 55.13 & 58.99 \\
\hline Vit. $B_{1}$ & 74.44 & 71.33 & 71.17 & 63.38 & 70.08 \\
\hline Vit. C & 75.56 & 75.42 & 70.87 & 68.38 & 72.56 \\
\hline Vit. E & 73.33 & 70.92 & 69.67 & 67.76 & 70.42 \\
\hline Vit. $B_{1}+$ Vit. $C+$ Vit. E & 84.67 & 78.40 & 77.83 & 77.24 & 79.54 \\
\hline Yeast & 86.00 & 80.00 & 79.43 & 78.53 & 80.99 \\
\hline Mean (A) & 76.04 & 72.65 & 71.29 & 68.40 & 72.09 \\
\hline L.S.D. at $5 \%$ & \multicolumn{2}{|c|}{ A : 1.78} & B : 1.58 & \multicolumn{2}{|r|}{$\mathrm{AB}: 3.16$} \\
\hline \multicolumn{6}{|c|}{$2^{\text {nd }}$ Cut } \\
\hline Control & 63.89 & 62.42 & 54.67 & 53.05 & 58.51 \\
\hline Vit. $B_{1}$ & 69.93 & 66.67 & 65.58 & 62.95 & 66.28 \\
\hline Vit. C & 73.14 & 69.13 & 66.44 & 65.83 & 68.64 \\
\hline Vit. E & 67.00 & 66.34 & 65.20 & 63.14 & 65.42 \\
\hline Vit. $B_{1}+$ Vit. C+Vit. E & 80.11 & 76.87 & 76.33 & 75.33 & 77.16 \\
\hline Yeast & 83.08 & 81.40 & 80.89 & 79.91 & 81.32 \\
\hline Mean (A) & 72.86 & 70.47 & 68.19 & 66.70 & 69.55 \\
\hline L.S.D. at $5 \%$ & \multicolumn{2}{|c|}{$\mathrm{A}: 1.17$} & B : 1.74 & \multicolumn{2}{|r|}{$\mathrm{AB}: 3.48$} \\
\hline \multicolumn{6}{|c|}{$3^{\text {rd }}$ Cut } \\
\hline Control & 62.22 & 60.25 & 52.40 & 47.48 & 55.59 \\
\hline Vit. $B_{1}$ & 64.80 & 64.50 & 63.44 & 53.38 & 61.53 \\
\hline Vit. C & 64.25 & 64.11 & 62.40 & 55.05 & 61.45 \\
\hline Vit. E & 64.25 & 63.22 & 60.87 & 53.76 & 60.53 \\
\hline Vit. $B_{1}+$ Vit. C+Vit. E & 77.00 & 73.33 & 69.52 & 68.27 & 72.03 \\
\hline Yeast & 79.17 & 78.00 & 76.47 & 73.24 & 76.72 \\
\hline Mean (A) & 68.62 & 67.24 & 64.18 & 58.53 & 64.64 \\
\hline L.S.D. at $5 \%$ & \multicolumn{2}{|c|}{ A : 2.25} & B : 2.36 & \multicolumn{2}{|r|}{$\mathrm{AB}: 4.72$} \\
\hline
\end{tabular}


Table 3. Effect of plant densities and some vitamins and yeast treatments on number of branches/plant of sweet basil (Ocimum basilicum, L.) plants during the first season.

\begin{tabular}{|c|c|c|c|c|c|}
\hline \multicolumn{6}{|c|}{$1^{\text {st }}$ Cut } \\
\hline \multirow{2}{*}{ Treatments } & \multicolumn{5}{|c|}{ Plant densities $/ 14.5 \mathrm{~m}^{2}$ (A) } \\
\hline & 145 & 105 & 80 & 65 & Mean (B) \\
\hline Control & 12.05 & 21.33 & 21.50 & 28.89 & 20.94 \\
\hline Vit. $B_{1}$ & 20.27 & 25.07 & 32.00 & 33.45 & 27.70 \\
\hline Vit. C & 25.38 & 26.73 & 32.75 & 34.89 & 29.94 \\
\hline Vit. E & 20.47 & 27.40 & 32.00 & 34.67 & 28.64 \\
\hline Vit. $B_{1}+$ Vit. C+Vit. E & 30.62 & 35.67 & 36.67 & 44.22 & 36.80 \\
\hline Yeast & 31.48 & 35.33 & 36.53 & 45.22 & 37.14 \\
\hline Mean (A) & 23.38 & 28.59 & 31.91 & 36.89 & 30.19 \\
\hline L.S.D. at $5 \%$ & \multicolumn{2}{|c|}{ A $: 1.57$} & B : 1.41 & \multicolumn{2}{|r|}{$\mathrm{AB}: 2.82$} \\
\hline \multicolumn{6}{|c|}{$2^{\text {nd }}$ Cut } \\
\hline Control & 19.75 & 27.00 & 27.75 & 36.22 & 58.09 \\
\hline Vit. $B_{1}$ & 29.72 & 32.13 & 38.42 & 45.56 & 36.46 \\
\hline Vit. C & 33.43 & 34.93 & 40.67 & 48.67 & 39.43 \\
\hline Vit. E & 32.67 & 36.07 & 38.25 & 48.00 & 26.75 \\
\hline Vit. $B_{1}+$ Vit. C+Vit. E & 36.57 & 43.67 & 46.83 & 60.89 & 46.99 \\
\hline Yeast & 38.76 & 45.67 & 46.75 & 58.73 & 47.48 \\
\hline Mean (A) & 31.82 & 36.58 & 39.78 & 49.68 & 39.46 \\
\hline L.S.D. at $5 \%$ & \multicolumn{2}{|c|}{ A : 1.55} & B : 1.08 & \multicolumn{2}{|r|}{$\mathrm{AB}: 2.16$} \\
\hline \multicolumn{6}{|c|}{$3^{\text {rd }}$ Cut } \\
\hline Control & 28.50 & 32.80 & 34.08 & 48.11 & 35.87 \\
\hline Vit. $B_{1}$ & 46.73 & 47.03 & 54.00 & 60.67 & 52.11 \\
\hline Vit. C & 49.86 & 51.33 & 54.25 & 66.00 & 55.36 \\
\hline Vit. E & 49.62 & 51.20 & 51.75 & 65.33 & 54.48 \\
\hline Vit. $B_{1}+$ Vit. C+Vit. E & 56.47 & 57.53 & 67.00 & 81.89 & 65.72 \\
\hline Yeast & 59.95 & 61.53 & 67.58 & 80.11 & 67.29 \\
\hline Mean (A) & 48.57 & 50.19 & 54.78 & 67.02 & 55.14 \\
\hline L.S.D. at $5 \%$ & \multicolumn{2}{|c|}{ A $: 1.72$} & B : 2.58 & \multicolumn{2}{|r|}{$\mathrm{AB}: 5.15$} \\
\hline
\end{tabular}


Table 4. Effect of plant densities and some vitamins and yeast treatments on number of branches/plant of sweet basil (Ocimum basilicum, L.) plants during the second season.

\begin{tabular}{|c|c|c|c|c|c|}
\hline \multicolumn{6}{|c|}{$1^{\text {st }}$ Cut } \\
\hline \multirow{2}{*}{ Treatments } & \multicolumn{5}{|c|}{ Plant densities $/ 14.5 \mathrm{~m}^{2}$ (A) } \\
\hline & 145 & 105 & 80 & 65 & Mean (B) \\
\hline Control & 19.00 & 19.95 & 22.83 & 25.11 & 21.72 \\
\hline Vit. $B_{1}$ & 24.72 & 26.00 & 26.27 & 33.45 & 27.61 \\
\hline Vit. C & 25.67 & 29.67 & 32.58 & 35.00 & 30.73 \\
\hline Vit. E & 24.19 & 26.87 & 28.00 & 33.11 & 28.04 \\
\hline Vit. $B_{1}+$ Vit. C +Vit. E & 32.38 & 34.73 & 36.50 & 38.18 & 35.45 \\
\hline Yeast & 33.67 & 35.60 & 38.17 & 40.22 & 36.92 \\
\hline Mean (A) & 26.61 & 28.80 & 30.73 & 34.18 & 30.08 \\
\hline L.S.D. at 5 \% & \multicolumn{2}{|c|}{ A : 1.03} & B : 1.02 & \multicolumn{2}{|r|}{$\mathrm{AB}: 2.04$} \\
\hline \multicolumn{6}{|c|}{$2^{\text {nd }}$ Cut } \\
\hline Control & 23.00 & 24.07 & 24.25 & 34.56 & 26.47 \\
\hline Vit. $B_{1}$ & 31.33 & 31.67 & 32.58 & 44.00 & 34.90 \\
\hline Vit. C & 32.33 & 34.33 & 37.17 & 47.11 & 37.74 \\
\hline Vit. E & 30.90 & 33.87 & 34.75 & 44.11 & 35.91 \\
\hline Vit. $B_{1}+$ Vit. C+Vit. E & 41.91 & 47.33 & 48.58 & 53.00 & 47.71 \\
\hline Yeast & 48.32 & 48.33 & 50.00 & 55.78 & 50.61 \\
\hline Mean (A) & 34.63 & 36.60 & 37.89 & 46.43 & 38.89 \\
\hline L.S.D. at $5 \%$ & \multicolumn{2}{|c|}{ A : 1.23} & B : 1.74 & \multicolumn{2}{|r|}{$\mathrm{AB}: 3.48$} \\
\hline \multicolumn{6}{|c|}{$3^{\text {rd }}$ Cut } \\
\hline Control & 22.57 & 29.60 & 31.42 & 45.22 & 32.20 \\
\hline Vit. $B_{1}$ & 38.52 & 39.47 & 46.00 & 61.00 & 46.25 \\
\hline Vit. C & 38.81 & 41.67 & 51.33 & 64.00 & 48.95 \\
\hline Vit. E & 36.62 & 40.00 & 50.33 & 63.11 & 47.52 \\
\hline Vit. $B_{1}+$ Vit. C+Vit. E & 57.91 & 60.87 & 64.75 & 75.11 & 64.66 \\
\hline Yeast & 62.57 & 64.80 & 69.00 & 76.33 & 68.18 \\
\hline Mean (A) & 42.83 & 46.07 & 52.14 & 64.13 & 51.29 \\
\hline L.S.D. at $5 \%$ & \multicolumn{2}{|c|}{ A : 2.69} & B : 2.68 & \multicolumn{2}{|r|}{ AB : N.S. } \\
\hline
\end{tabular}


M.A.H. Abdou et al.

Table 5. Effect of plant densities and some vitamins and yeast treatments on leaf area $\left(\mathrm{cm}^{2}\right)$ of sweet basil (Ocimum basilicum, L.) plants during the first season.

\begin{tabular}{|c|c|c|c|c|c|}
\hline \multicolumn{6}{|c|}{$1^{\text {st }}$ Cut } \\
\hline \multirow{2}{*}{ Treatments } & \multicolumn{5}{|c|}{ Plant densities $/ 14.5 \mathrm{~m}^{2}$ (A) } \\
\hline & 145 & 105 & 80 & 65 & Mean (B) \\
\hline Control & 3.55 & 3.92 & 5.56 & 6.06 & 4.77 \\
\hline Vit. $B_{1}$ & 6.48 & 6.51 & 8.05 & 8.06 & 7.28 \\
\hline Vit. C & 6.71 & 7.05 & 8.13 & 8.81 & 7.68 \\
\hline Vit. E & 6.40 & 6.48 & 8.65 & 9.57 & 7.78 \\
\hline Vit. $B_{1}+$ Vit. C+Vit. E & 8.98 & 9.33 & 10.03 & 13.02 & 10.34 \\
\hline Yeast & 9.27 & 9.77 & 11.63 & 13.66 & 11.08 \\
\hline Mean (A) & 6.90 & 7.18 & 8.68 & 9.86 & 8.15 \\
\hline L.S.D. at $5 \%$ & \multicolumn{2}{|c|}{ A : 1.97} & B : 1.09 & \multicolumn{2}{|r|}{$A B:$ N.S. } \\
\hline \multicolumn{6}{|c|}{$2^{\text {nd }}$ Cut } \\
\hline Control & 4.75 & 5.68 & 5.70 & 5.99 & 5.53 \\
\hline Vit. $B_{1}$ & 7.94 & 8.47 & 9.81 & 10.44 & 9.17 \\
\hline Vit. C & 8.30 & 8.96 & 9.73 & 12.03 & 9.76 \\
\hline Vit. E & 8.07 & 8.59 & 10.52 & 11.17 & 9.59 \\
\hline Vit. $B_{1}+$ Vit. C+Vit. E & 11.11 & 12.03 & 13.42 & 14.42 & 12.75 \\
\hline Yeast & 13.60 & 14.18 & 15.39 & 15.61 & 14.70 \\
\hline Mean (A) & 8.96 & 9.65 & 10.76 & 11.61 & 10.25 \\
\hline L.S.D. at $5 \%$ & \multicolumn{2}{|c|}{ A : 1.28} & B : 1.24 & \multicolumn{2}{|r|}{ AB : N.S. } \\
\hline \multicolumn{6}{|c|}{$3^{\text {rd }}$ Cut } \\
\hline Control & 4.97 & 5.06 & 5.14 & 5.33 & 5.13 \\
\hline Vit. $B_{1}$ & 6.87 & 7.20 & 7.99 & 8.53 & 7.65 \\
\hline Vit. C & 7.31 & 8.62 & 9.35 & 9.66 & 8.74 \\
\hline Vit. E & 7.79 & 7.98 & 8.53 & 8.93 & 8.31 \\
\hline Vit. $B_{1}+$ Vit. C+Vit. E & 10.29 & 12.05 & 13.53 & 13.68 & 12.39 \\
\hline Yeast & 10.63 & 14.40 & 14.60 & 14.63 & 13.57 \\
\hline Mean (A) & 7.98 & 9.22 & 9.86 & 10.13 & 9.29 \\
\hline L.S.D. at $5 \%$ & \multicolumn{2}{|c|}{ A : 0.69} & B : 0.93 & \multicolumn{2}{|r|}{$\mathrm{AB}: 1.86$} \\
\hline
\end{tabular}


Table 6. Effect of plant densities and some vitamins and yeast treatments on leaf area $\left(\mathrm{cm}^{2}\right)$ of sweet basil (Ocimum basilicum, L.) plants during the second season.

\begin{tabular}{|c|c|c|c|c|c|}
\hline \multicolumn{6}{|c|}{$1^{\text {st }}$ Cut } \\
\hline \multirow{2}{*}{ Treatments } & \multicolumn{5}{|c|}{ Plant densities $/ 14.5 \mathrm{~m}^{2}$ (A) } \\
\hline & 145 & 105 & 80 & 65 & Mean (B) \\
\hline Control & 5.50 & 5.55 & 5.61 & 5.70 & 5.59 \\
\hline Vit. $B_{1}$ & 7.85 & 8.02 & 8.30 & 9.12 & 8.32 \\
\hline Vit. C & 8.35 & 9.21 & 10.30 & 10.58 & 9.61 \\
\hline Vit. E & 7.58 & 7.66 & 8.55 & 10.13 & 8.48 \\
\hline Vit. $B_{1}+$ Vit. $C+$ Vit. E & 10.81 & 11.24 & 11.80 & 12.67 & 11.63 \\
\hline Yeast & 10.79 & 11.54 & 14.45 & 15.71 & 13.12 \\
\hline Mean (A) & 8.48 & 8.87 & 9.84 & 10.65 & 9.46 \\
\hline L.S.D. at $5 \%$ & \multicolumn{2}{|c|}{ A : 0.61} & B : 0.93 & \multicolumn{2}{|r|}{$\mathrm{AB}: 1.87$} \\
\hline \multicolumn{6}{|c|}{$2^{\text {nd }}$ Cut } \\
\hline Control & 4.74 & 4.75 & 5.24 & 5.29 & 5.01 \\
\hline Vit. $B_{1}$ & 7.00 & 8.50 & 10.29 & 10.59 & 9.10 \\
\hline Vit. C & 7.49 & 8.53 & 9.63 & 10.02 & 8.92 \\
\hline Vit. E & 7.27 & 7.73 & 9.61 & 10.17 & 8.70 \\
\hline Vit. $B_{1}+$ Vit. C+Vit. E & 9.79 & 11.27 & 11.73 & 12.77 & 11.39 \\
\hline Yeast & 10.21 & 11.11 & 12.16 & 12.71 & 11.55 \\
\hline Mean (A) & 7.75 & 8.65 & 9.78 & 10.26 & 9.11 \\
\hline L.S.D. at $5 \%$ & \multicolumn{2}{|c|}{$A: 0.30$} & B : 0.50 & \multicolumn{2}{|r|}{ AB : 1.00} \\
\hline \multicolumn{6}{|c|}{$3^{\text {rd }}$ Cut } \\
\hline Control & 4.84 & 4.89 & 6.06 & 6.06 & 5.46 \\
\hline Vit. $B_{1}$ & 8.52 & 10.41 & 10.71 & 11.82 & 10.37 \\
\hline Vit. C & 8.27 & 10.33 & 10.40 & 11.84 & 10.21 \\
\hline Vit. E & 8.60 & 10.43 & 10.74 & 11.94 & 10.43 \\
\hline Vit. $B_{1}+$ Vit. C+Vit. E & 10.58 & 12.74 & 13.34 & 13.50 & 12.54 \\
\hline Yeast & 11.02 & 12.46 & 14.41 & 14.66 & 13.14 \\
\hline Mean (A) & 8.64 & 10.21 & 10.94 & 11.64 & 10.36 \\
\hline L.S.D. at 5 \% & \multicolumn{2}{|c|}{ A : 0.46} & B : 0.41 & \multicolumn{2}{|r|}{$\mathrm{AB}: 0.82$} \\
\hline
\end{tabular}


Table 7. Effect of plant densities and some vitamins and yeast treatments on herb fresh weight/plant/cut (g/plant) of sweet basil (Ocimum basilicum, L.) plants during the first season.

\begin{tabular}{|c|c|c|c|c|c|}
\hline \multicolumn{6}{|c|}{$1^{\text {st }}$ Cut } \\
\hline \multirow{2}{*}{ Treatments } & \multicolumn{5}{|c|}{ Plant densities $/ 14.5 \mathrm{~m}^{2}$ (A) } \\
\hline & 145 & 105 & 80 & 65 & Mean (B) \\
\hline Control-10 & 83.6 & 153.0 & 167.5 & 190.6 & 148.7 \\
\hline Vit. $B_{1}$ & 194.5 & 227.9 & 236.3 & 242.8 & 225.4 \\
\hline Vit. C & 243.0 & 246.7 & 270.6 & 289.6 & 262.5 \\
\hline Vit. E & 282.7 & 249.3 & 253.3 & 271.7 & 264.3 \\
\hline Vit. $B_{1}+$ Vit. C+Vit. E & 283.6 & 330.8 & 333.9 & 349.2 & 323.5 \\
\hline Yeast & 330.7 & 348.8 & 350.3 & 355.0 & 346.7 \\
\hline Mean (A) & 239.4 & 256.2 & 268.7 & 283.2 & 261.8 \\
\hline L.S.D. at $5 \%$ & \multicolumn{2}{|c|}{ A : 10.1} & B : 12.1 & \multicolumn{2}{|r|}{$\mathrm{AB}: 24.2$} \\
\hline \multicolumn{6}{|c|}{$2^{\text {nd }}$ Cut } \\
\hline Control & 145.1 & 169.7 & 185.0 & 235.6 & 183.8 \\
\hline Vit. $B_{1}$ & 208.6 & 283.0 & 285.0 & 340.0 & 279.1 \\
\hline Vit. C & 181.3 & 309.0 & 353.8 & 383.9 & 307.0 \\
\hline Vit. E & 182.4 & 301.3 & 313.3 & 376.1 & 293.3 \\
\hline Vit. $B_{1}+$ Vit. C+Vit. E & 379.2 & 426.7 & 450.3 & 477.8 & 433.5 \\
\hline Yeast & 235.9 & 431.3 & 469.0 & 478.7 & 453.7 \\
\hline Mean (A) & 255.4 & 320.2 & 342.7 & 382.0 & 322.6 \\
\hline L.S.D. at $5 \%$ & \multicolumn{2}{|c|}{ A : 40.7} & B : 40.6 & \multicolumn{2}{|r|}{$\mathrm{AB}: 81.2$} \\
\hline \multicolumn{6}{|c|}{$3^{\text {rd }}$ Cut } \\
\hline Control & 309.7 & 241.1 & 245.0 & 253.0 & 262.2 \\
\hline Vit. $B_{1}$ & 313.2 & 394.7 & 395.8 & 466.7 & 392.6 \\
\hline Vit. C & 324.3 & 405.0 & 434.3 & 570.6 & 433.5 \\
\hline Vit. E & 309.5 & 382.9 & 394.3 & 557.2 & 411.0 \\
\hline Vit. $B_{1}+$ Vit. C+Vit. E & 488.5 & 497.0 & 510.8 & 645.0 & 535.3 \\
\hline Yeast & 553.6 & 534.3 & 559.6 & 647.0 & 573.6 \\
\hline Mean (A) & 383.1 & 409.2 & 423.3 & 523.3 & 434.3 \\
\hline L.S.D. at $5 \%$ & \multicolumn{2}{|c|}{ A : 23.1} & B : 16.9 & \multicolumn{2}{|r|}{$\mathrm{AB}: 33.8$} \\
\hline
\end{tabular}


Table 8. Effect of plant densities and some vitamins and yeast treatments on herb fresh weight/plant/cut (g/plant) of sweet basil (Ocimum basilicum, L.) plants during the second season.

\begin{tabular}{|c|c|c|c|c|c|}
\hline \multicolumn{6}{|c|}{$1^{\text {st }}$ Cut } \\
\hline \multirow{2}{*}{ Treatments } & \multicolumn{5}{|c|}{ Plant densities $/ 14.5 \mathrm{~m}^{2}$ (A) } \\
\hline & 145 & 105 & 80 & 65 & Mean (B) \\
\hline Control & 156.5 & 147.3 & 162.8 & 168.6 & 158.8 \\
\hline Vit. $B_{1}$ & 216.3 & 237.7 & 244.6 & 258.9 & 239.4 \\
\hline Vit. C & 205.5 & 254.3 & 258.8 & 260.6 & 244.8 \\
\hline Vit. E & 209.7 & 243.0 & 247.1 & 255.0 & 238.7 \\
\hline Vit. $B_{1}+$ Vit. $C+$ Vit. E & 282.3 & 267.0 & 287.1 & 312.2 & 287.2 \\
\hline Yeast & 293.6 & 272.3 & 291.3 & 313.9 & 292.8 \\
\hline Mean (A) & 227.3 & 236.9 & 248.6 & 261.5 & 243.6 \\
\hline L.S.D. at $5 \%$ & \multicolumn{2}{|c|}{ A : 6.2} & B : 9.4 & \multicolumn{2}{|r|}{$\mathrm{AB}: 18.8$} \\
\hline \multicolumn{6}{|c|}{$2^{\text {nd }}$ Cut } \\
\hline Control & 201.9 & 211.0 & 237.5 & 251.1 & 225.4 \\
\hline Vit. $B_{1}$ & 307.0 & 329.0 & 337.1 & 350.0 & 330.8 \\
\hline Vit. C & 300.9 & 353.0 & 356.7 & 363.3 & 343.5 \\
\hline Vit. E & 290.0 & 343.8 & 347.0 & 363.3 & 336.0 \\
\hline Vit. $B_{1}+$ Vit. C+Vit. E & 455.9 & 469.2 & 469.7 & 476.7 & 467.9 \\
\hline Yeast & 499.6 & 519.3 & 551.7 & 552.1 & 530.7 \\
\hline Mean (A) & 342.5 & 370.9 & 383.3 & 392.8 & 372.4 \\
\hline L.S.D. at $5 \%$ & \multicolumn{2}{|c|}{ A : 7.5} & B : 20.4 & \multicolumn{2}{|r|}{$\mathrm{AB}: 40.8$} \\
\hline \multicolumn{6}{|c|}{$3^{\text {rd }}$ Cut } \\
\hline Control & 291.4 & 238.8 & 247.7 & 297.2 & 268.8 \\
\hline Vit. $B_{1}$ & 358.3 & 354.3 & 375.0 & 460.6 & 387.1 \\
\hline Vit. C & 381.1 & 376.3 & 383.3 & 478.3 & 404.8 \\
\hline Vit. E & 372.6 & 363.7 & 371.4 & 462.8 & 392.6 \\
\hline Vit. $B_{1}+$ Vit. C+Vit. E & 536.3 & 550.3 & 585.0 & 603.3 & 568.7 \\
\hline Yeast & 591.0 & 575.7 & 598.8 & 641.7 & 601.8 \\
\hline Mean (A) & 421.8 & 409.9 & 426.9 & 490.7 & 437.3 \\
\hline L.S.D. at 5 \% & \multicolumn{2}{|c|}{ A : 26.5} & B : 17.9 & \multicolumn{2}{|r|}{$\mathrm{AB}: 35.8$} \\
\hline
\end{tabular}


Table 9. Effect of plant densities and some vitamins and yeast treatments on herb dry weight/plant/cut (g/plant) of sweet basil (Ocimum basilicum, L.) plants during the first season.

\begin{tabular}{|c|c|c|c|c|c|}
\hline \multicolumn{6}{|c|}{$1^{\text {st }}$ Cut } \\
\hline \multirow{2}{*}{ Treatments } & \multicolumn{5}{|c|}{ Plant densities $/ 14.5 \mathrm{~m}^{2}$ (A) } \\
\hline & 145 & 105 & 80 & 65 & Mean (B) \\
\hline Control & 33.58 & 32.13 & 42.83 & 50.00 & 39.64 \\
\hline Vit. $B_{1}$ & 39.16 & 53.47 & 58.83 & 60.00 & 52.86 \\
\hline Vit. C & 44.10 & 60.40 & 62.17 & 71.11 & 59.45 \\
\hline Vit. E & 41.48 & 57.60 & 60.50 & 59.78 & 54.84 \\
\hline Vit. $B_{1}+$ Vit. C+Vit. E & 67.05 & 76.13 & 88.50 & 89.78 & 80.36 \\
\hline Yeast & 72.81 & 82.00 & 96.17 & 102.00 & 88.24 \\
\hline Mean (A) & 49.70 & 60.29 & 68.17 & 72.11 & 62.57 \\
\hline L.S.D. at $5 \%$ & A : 15.69 & & B : 9.61 & & 19.22 \\
\hline \multicolumn{6}{|c|}{$2^{\text {nd }}$ Cut } \\
\hline Control & 36.42 & 35.20 & 44.83 & 73.11 & 47.39 \\
\hline Vit. $B_{1}$ & 46.37 & 61.73 & 73.10 & 94.44 & 68.91 \\
\hline Vit. C & 49.68 & 60.53 & 73.67 & 103.51 & 71.85 \\
\hline Vit. E & 48.21 & 62.67 & 69.50 & 109.78 & 72.54 \\
\hline Vit. $B_{1}+$ Vit. C+Vit. E & 67.99 & 112.93 & 116.00 & 128.22 & 106.29 \\
\hline Yeast & 77.21 & 111.11 & 129.50 & 130.93 & 112.19 \\
\hline Mean (A) & 54.31 & 74.03 & 84.43 & 106.67 & 79.86 \\
\hline L.S.D. at $5 \%$ & A : 10.28 & & B : 26.83 & & 53.66 \\
\hline \multicolumn{6}{|c|}{$3^{\text {rd }}$ Cut } \\
\hline Control & 49.76 & 58.67 & 59.20 & 75.56 & 60.80 \\
\hline Vit. $B_{1}$ & 56.40 & 86.40 & 90.17 & 136.66 & 92.41 \\
\hline Vit. C & 73.12 & 91.47 & 96.50 & 148.22 & 102.33 \\
\hline Vit. E & 62.44 & 88.27 & 89.50 & 144.44 & 96.16 \\
\hline Vit. $B_{1}+$ Vit. C+Vit. E & 134.72 & 137.47 & 138.56 & 146.83 & 138.40 \\
\hline Yeast & 136.80 & 123.87 & 178.00 & 200.00 & 159.67 \\
\hline Mean (A) & 84.87 & 97.69 & 108.66 & 141.95 & 108.29 \\
\hline L.S.D. at $5 \%$ & A : 24.51 & & B : 18.18 & & 36.36 \\
\hline
\end{tabular}


Table 10. Effect of plant densities and some vitamins (vit. $B_{1}$, vit. $C$ and vit. $E$ ) and yeast treatments on herb dry weight/plant/cut (g/plant) of sweet basil (Ocimum basilicum, $\mathrm{L}$.) plants during the second season.

\begin{tabular}{|c|c|c|c|c|c|}
\hline \multicolumn{6}{|c|}{$\mathbf{1}^{\text {st }}$ Cut } \\
\hline \multirow{2}{*}{ Treatments } & \multicolumn{5}{|c|}{ Plant densities $/ 14.5 \mathrm{~m}^{2}$ (A) } \\
\hline & 145 & 105 & 80 & 65 & Mean (B) \\
\hline Control & 38.19 & 18.93 & 48.67 & 55.11 & 40.22 \\
\hline Vit. $B_{1}$ & 41.62 & 55.33 & 57.17 & 59.11 & 53.31 \\
\hline Vit. C & 44.95 & 63.20 & 64.33 & 78.67 & 62.79 \\
\hline Vit. E & 43.14 & 55.87 & 58.67 & 60.27 & 54.49 \\
\hline Vit. $B_{1}+$ Vit. $C+$ Vit. E & 52.00 & 83.47 & 87.33 & 90.89 & 78.42 \\
\hline Yeast & 62.48 & 88.27 & 95.83 & 103.33 & 87.48 \\
\hline Mean (A) & 23.06 & 68.84 & 76.67 & 82.56 & 62.78 \\
\hline L.S.D. at $5 \%$ & \multicolumn{2}{|c|}{ A : 6.02} & B : 8.47 & \multicolumn{2}{|r|}{ AB : 16.94} \\
\hline \multicolumn{6}{|c|}{$2^{\text {nd }} \mathrm{Cut}$} \\
\hline Control & 54.76 & 48.00 & 55.50 & 64.22 & 55.62 \\
\hline Vit. $B_{1}$ & 64.19 & 89.47 & 91.83 & 98.22 & 85.93 \\
\hline Vit. C & 68.67 & 94.13 & 99.33 & 103.72 & 91.46 \\
\hline Vit. E & 65.43 & 91.20 & 91.50 & 102.44 & 87.64 \\
\hline Vit. $B_{1}+$ Vit. C+Vit. E & 104.28 & 134.50 & 138.40 & 139.11 & 129.08 \\
\hline Yeast & 110.48 & 151.23 & 159.50 & 169.33 & 147.64 \\
\hline Mean (A) & 53.97 & 109.42 & 114.01 & 120.84 & 99.56 \\
\hline L.S.D. at $5 \%$ & \multicolumn{2}{|c|}{ A : 26.39} & B : 20.44 & \multicolumn{2}{|r|}{$\mathrm{AB}: 40.88$} \\
\hline \multicolumn{6}{|c|}{$3^{\text {rd }}$ Cut } \\
\hline Control & 61.62 & 62.45 & 71.67 & 82.00 & 69.44 \\
\hline Vit. $B_{1}$ & 97.05 & 116.80 & 126.17 & 132.44 & 118.12 \\
\hline Vit. C & 104.90 & 121.60 & 132.67 & 144.22 & 125.85 \\
\hline Vit. E & 99.81 & 119.20 & 129.00 & 136.22 & 121.06 \\
\hline Vit. $B_{1}+$ Vit. C+Vit. E & 150.57 & 169.50 & 177.07 & 181.56 & 169.68 \\
\hline Yeast & 144.00 & 204.27 & 209.00 & 218.00 & 193.82 \\
\hline Mean (A) & 109.66 & 140.30 & 148.93 & 157.08 & 131.82 \\
\hline L.S.D. at $5 \%$ & \multicolumn{2}{|c|}{ A : 31.71} & B : 17.37 & \multicolumn{2}{|r|}{$\mathrm{AB}: 34.74$} \\
\hline
\end{tabular}


The treatment of vit. $\mathrm{B}_{1}+$ vit. $\mathrm{C}+$ vit. $\mathrm{E}$ resulted the next order in this concern.

Addition of active yeast to the plants significantly increased plant growth parameters as revealed by Salman (2006); El-Keasy et al. (2011); Abd El-Salam (2014) and Nassar et al. (2015) on basil plant. The positive effect of vitamins on improving plant growth was reported by Khalil et al. (2010) and Abd El-Salam (2014) on sweet basil.

The interaction between main and sub plot $(\mathrm{A} \times \mathrm{B})$ treatments was significant for number of branches, leaf area, herb fresh and dry weights/plant/cut during the three cuts in both seasons, except for number of branches in the third cut in the second season and leaf area during the second cut in the first season as clearly shown in Tables (3 to 10). The best interaction treatments were obtained by 65 plants $/ 14.5 \mathrm{~m}^{2}$ in combination with active yeast or vit. $\mathrm{B}_{1}+$ vit. $\mathrm{C}+$ vit. $\mathrm{E}$.

\section{Herb fresh and dry weights/plant/season and /fed/season:}

Data presented in Tables (11 to 14) indicated that the effect of density on herb fresh and dry weights/plant/season and /fed/season becomes significant. Comparing the means showed that density of 65 plants $/ 14.5 \mathrm{~m}^{2}$ had a higher values of herb fresh and dry weights/plant compared to the other treatments. Moreover, both characters of herb fresh and dry weights/fed were the highest values with the highest density (40000 plants/fed) as compared with other densities (28966, 22069, 17931 plants/fed).

The number of plants per unit area is the most important among yield components (Khafi, 2003).

Similar results were obtained by Balyan and Sobti (1990); Davis (1993) and Chegini et al. (2012) on sweet basil.

Data, also, sweet basil that all used five treatments of vitamins and active yeast significantly increased herb fresh and dry weights/plant and /fed in both seasons comparing with control treatments. The treatment of active yeast recorded the maximum yield herb fresh (34.2 and 35.5 ton/fed in both seasons, respectively) and dry weights (9.336 and 11.146 ton/fed in both seasons, respectively) as compared with other treatments.

Many investigators proved that treated plants with active yeast led to significantly increased in total fresh and dry weights such as, Salman (2006) and Abd El-Salam (2014) on Ocimum basilicum, Massoud (2006) and Mosaad (2012) on sage plants. Concerning the effect of vitamins, Abd El-Salam (2014) on basil plants and Abd El-Naeem (2012) on mint plants found that vitamins treatments had positive effect on herb fresh and dry weights.

The interaction between main and sub plots $(A \times B)$ was significant for herb fresh and dry weights/plant and /fed in both seasons (Tables, 11, 12, 13 and 14). The best interaction treatments for herb fresh and dry weights/plant were lowest density (65 plants $/ 14.5 \mathrm{~m}^{2}$ ) with active yeast or vit. $\mathrm{B}_{1}+$ vit. C + vit. E (Tables, 11 and 12).

The highest values for yield/fed were obtained by the highest density (145 plants per $14.5 \mathrm{~m}^{2}$ ) in combination with active yeast or vit. $B_{1}+$ vit. $C+$ vit. $E$.

\section{REFERENCES}

Abbas, M.S. (2014). Assessment of density and cultivation type on growth and yield of two cultivars of basil (Ocimum basilucum, L.). Int. J. Agronomy \& Agric. Res., 5(1):74-79.

Abd El-Naeem, L.M.A. (2012). Physiological Studies on Mint Plants. Ph.D. Thesis, Fac. Agric., Minia Univ., Egypt.

Abd El-Salam, Nora M.K. (2014). Response of Sweet Basil Plants to Some Agricultural Treatments. Ph.D. Thesis, Fac. Agric., Minia Univ.

Atghaei, M.S.; Rajabi, A. and Hemayaty, S.S. (2015). Investigating the effect of bush density on the yield and leaf essence 
Table 11. Effect of plant densities and some vitamins and yeast treatments on total herb fresh weight/plant/season (kg/plant) of sweet basil (Ocimum basilicum, L.) plants.

\begin{tabular}{|c|c|c|c|c|c|}
\hline \multicolumn{6}{|c|}{ First season } \\
\hline \multirow{2}{*}{ Treatments } & \multicolumn{5}{|c|}{ Plant densities /14.5 $\mathrm{m}^{2}$ (A) } \\
\hline & 145 & 105 & 80 & 65 & Mean (B) \\
\hline Control & 0.538 & 0.564 & 0.598 & 0.679 & 0.595 \\
\hline Vit. $B_{1}$ & 0.716 & 0.906 & 0.917 & 1.050 & 0.897 \\
\hline Vit. C & 0.749 & 0.961 & 1.059 & 1.244 & 1.003 \\
\hline Vit. E & 0.775 & 0.934 & 0.961 & 1.205 & 0.969 \\
\hline Vit. $B_{1}+$ Vit. C+Vit. E & 1.081 & 1.251 & 1.295 & 1.472 & 1.275 \\
\hline Yeast & 1.338 & 1.298 & 1.379 & 1.481 & 1.374 \\
\hline Mean (A) & 0.866 & 0.986 & 1.035 & 1.188 & \\
\hline L.S.D. at $5 \%$ & \multicolumn{2}{|c|}{ A : 0.045} & B : 0.07 & \multicolumn{2}{|r|}{$\mathrm{AB}: 0.140$} \\
\hline \multicolumn{6}{|c|}{ Second season } \\
\hline Control & 0.650 & 0.597 & 0.648 & 0.717 & 0.653 \\
\hline Vit. $B_{1}$ & 0.882 & 0.921 & 0.957 & 1.070 & 0.957 \\
\hline Vit. C & 0.887 & 0.984 & 0.999 & 1.102 & 0.993 \\
\hline Vit. E & 0.872 & 0.951 & 0.966 & 1.081 & 0.967 \\
\hline Vit. $B_{1}+$ Vit. C+Vit. E & 1.275 & 1.287 & 1.342 & 1.392 & 1.324 \\
\hline Yeast & 1.384 & 1.367 & 1.442 & 1.508 & 1.425 \\
\hline Mean (A) & 0.992 & 1.018 & 1.059 & 1.145 & \\
\hline L.S.D. at $5 \%$ & \multicolumn{2}{|c|}{$A: 0.025$} & B : 0.06 & \multicolumn{2}{|r|}{$\mathrm{AB}: 0.122$} \\
\hline
\end{tabular}

Table 12. Effect of plant densities and some vitamins and yeast treatments on total herb dry weight/plant/season (kg/plant) of sweet basil (Ocimum basilicum, L.) plants.

\begin{tabular}{|c|c|c|c|c|c|}
\hline \multicolumn{6}{|c|}{ First season } \\
\hline \multirow{2}{*}{ Treatments } & \multicolumn{5}{|c|}{ Plant densities $/ 14.5 \mathrm{~m}^{2}$ (A) } \\
\hline & 145 & 105 & 80 & 65 & Mean (B) \\
\hline Control & 0.120 & 0.126 & 0.147 & 0.199 & 0.148 \\
\hline Vit. $B_{1}$ & 0.142 & 0.202 & 0.222 & 0.291 & 0.214 \\
\hline Vit. C & 0.167 & 0.212 & 0.232 & 0.323 & 0.234 \\
\hline Vit. E & 0.152 & 0.209 & 0.220 & 0.314 & 0.224 \\
\hline Vit. $B_{1}+$ Vit. C+Vit. E & 0.270 & 0.327 & 0.343 & 0.365 & 0.325 \\
\hline Yeast & 0.287 & 0.317 & 0.404 & 0.433 & 0.360 \\
\hline Mean (A) & 0.189 & 0.232 & 0.261 & 0.321 & \\
\hline L.S.D. at $5 \%$ & A : & & B : 0.034 & & $\mathrm{AB}: 0.068$ \\
\hline \multicolumn{6}{|c|}{ Second season } \\
\hline Control & 0.155 & 0.129 & 0.176 & 0.201 & 0.165 \\
\hline Vit. $B_{1}$ & 0.203 & 0.262 & 0.275 & 0.290 & 0.257 \\
\hline Vit. C & 0.219 & 0.279 & 0.296 & 0.327 & 0.280 \\
\hline Vit. E & 0.208 & 0.266 & 0.279 & 0.299 & 0.263 \\
\hline Vit. $B_{1}+$ Vit. C+Vit. E & 0.307 & 0.387 & 0.403 & 0.412 & 0.377 \\
\hline Yeast & 0.317 & 0.444 & 0.464 & 0.491 & 0.429 \\
\hline Mean (A) & 0.187 & 0.319 & 0.340 & 0.360 & \\
\hline L.S.D. at $5 \%$ & \multicolumn{2}{|c|}{ A : 0.017} & B : 0.023 & & $\mathrm{AB}: 0.046$ \\
\hline
\end{tabular}


Table 13. Effect of plant densities and some vitamins and yeast treatments on total herb fresh weight/fed/season (ton/fed) of sweet basil (Ocimum basilicum, L.) plants.

\begin{tabular}{|c|c|c|c|c|c|}
\hline \multicolumn{6}{|c|}{ First season } \\
\hline \multirow{2}{*}{ Treatments } & \multicolumn{5}{|c|}{ Plant densities /14.5 $\mathrm{m}^{2}$ (A) } \\
\hline & 145 & 105 & 80 & 65 & Mean (B) \\
\hline Control & 20.0 & 14.8 & 12.4 & 11.2 & 14.6 \\
\hline Vit. $B_{1}$ & 26.7 & 23.7 & 19.0 & 17.4 & 21.7 \\
\hline Vit. C & 27.9 & 25.2 & 21.9 & 20.6 & 23.9 \\
\hline Vit. E & 28.8 & 24.5 & 19.9 & 19.9 & 23.3 \\
\hline Vit. $B_{1}+$ Vit. C+Vit. E & 42.9 & 32.8 & 26.8 & 24.4 & 31.7 \\
\hline Yeast & 49.8 & 34.0 & 28.5 & 24.5 & 34.2 \\
\hline Mean (A) & 32.7 & 25.8 & 21.4 & 19.7 & \\
\hline L.S.D. at $5 \%$ & \multicolumn{2}{|c|}{$\mathrm{A}: 1.0$} & B : 0.9 & \multicolumn{2}{|r|}{$\mathrm{AB}: 1.8$} \\
\hline \multicolumn{6}{|c|}{ Second season } \\
\hline Control & 24.2 & 15.6 & 13.4 & 11.9 & 16.3 \\
\hline Vit. $B_{1}$ & 32.8 & 24.1 & 19.8 & 17.7 & 23.6 \\
\hline Vit. C & 33.0 & 25.8 & 20.7 & 18.2 & 24.4 \\
\hline Vit. E & 32.5 & 24.9 & 20.0 & 17.9 & 23.8 \\
\hline Vit. $B_{1}+$ Vit. C+Vit. E & 47.5 & 33.7 & 27.8 & 23.0 & 33.0 \\
\hline Yeast & 51.5 & 35.8 & 29.8 & 25.0 & 35.5 \\
\hline Mean (A) & 36.9 & 26.7 & 21.9 & 19.0 & \\
\hline L.S.D. at $5 \%$ & \multicolumn{2}{|c|}{$\mathrm{A}: 1.2$} & B : $2 . \mathrm{C}$ & \multicolumn{2}{|r|}{$\mathrm{AB}: 4.0$} \\
\hline
\end{tabular}

Table 14. Effect of plant densities and some vitamins and yeast treatments on total herb dry weight/fed/season (ton/fed) of sweet basil (Ocimum basilicum, L.) plants.

\begin{tabular}{|c|c|c|c|c|c|}
\hline \multicolumn{6}{|c|}{ First season } \\
\hline \multirow{2}{*}{ Treatments } & \multicolumn{5}{|c|}{ Plant densities $/ 14.5 \mathrm{~m}^{2}$ (A) } \\
\hline & 145 & 105 & 80 & 65 & Mean (B) \\
\hline$\overline{\text { Control }}$ & 4.800 & 3.650 & 3.244 & 3.568 & 3.816 \\
\hline Vit. $B_{1}$ & 5.680 & 5.851 & 4.899 & 5.218 & 5.412 \\
\hline Vit. $C$ & 6.680 & 6.141 & 5.120 & 5.792 & 5.933 \\
\hline Vit. E & 6.080 & 6.054 & 4.855 & 5.630 & 5.655 \\
\hline Vit. $B_{1}+$ Vit. C+Vit. E & 10.800 & 9.472 & 7.570 & 6.545 & 8.597 \\
\hline Yeast & 11.480 & 9.182 & 8.916 & 7.764 & 9.336 \\
\hline Mean (A) & 7.587 & 6.725 & 5.767 & 5.753 & \\
\hline L.S.D. at $5 \%$ & $\mathrm{~A}: 0$ & & B : 0.401 & & $\mathrm{AB}: 0.802$ \\
\hline \multicolumn{6}{|c|}{ Second season } \\
\hline Control & 6.200 & 3.737 & 3.884 & 3.604 & 4.356 \\
\hline Vit. $B_{1}$ & 8.120 & 7.589 & 6.069 & 5.200 & 6.745 \\
\hline Vit. C & 8.760 & 8.082 & 6.532 & 5.863 & 7.309 \\
\hline Vit. E & 8.320 & 7.705 & 6.157 & 5.361 & 6.886 \\
\hline Vit. $B_{1}+$ Vit. C+Vit. E & 12.280 & 11.209 & 8.894 & 7.388 & 9.943 \\
\hline Yeast & 12.680 & 12.861 & 10.240 & 8.804 & 11.146 \\
\hline Mean (A) & 9.393 & 8.531 & 6.963 & 6.037 & \\
\hline L.S.D. at $5 \%$ & \multicolumn{2}{|c|}{ A : 0.801} & B : 0.502 & & $\mathrm{AB}: 1.004$ \\
\hline
\end{tabular}


of green basil landraces (Ocimum basilicum). International Journal of Research Studies in Agricultural Sciences (IJRSAS), 1(1):11-17.

Balyan, S.; Pal, S.; Sharma, N.; Singh, S. and Sobti, S.N. (1987). Effect of spacing, nitrogen and phosphorus on the growth and yield of two Ocimum species. Indian performer, 31(2):89-96.

Balyan, S.S. and Sobti, S.N. (1990). Effect of nitrogen, phosphorus and potassium on dry matter accumulation and nutrient uptake pattern in Ocimum gratissimum (var. clocimum). Ind. Perumer, 34(3): 225-231 (Hort., Abst.; 61:8334).

Bekhradi, Delshad, M.; Kashi, A.; Babalar, M. and Ilkhani, S. (2014). Effect of plant density in some basil cultivars on yield and radiation use efficiency. J. Bio. \& Env. Sci., 5(1):91-96.

Botros, W.S.E. (2013). Physiological Studies on Caraway Plants. M.Sc. Thesis, Fac. Agric., Minia Univ. Egypt.

Chegini, N.; Zadeh, S.; Rad, A. and Bitarafan, Z. (2012). Study the nitrogen rate effect on sweet basil phonological stages and physiological indices under different plant densities and patterns. Int. J. Sci. \& Advanced Tech., 2(4):25-33.

Davis, J.M. (1993). Studies in row plant spacing and yield of fresh market basil. J. of Herbs Spices and Medicinal Plants, 2(1):35-43.

El-Keasy, W.A.; Aly, R.H. and Ibrahem, G.A. (2011). Influence of application of yeast and macerated black tea to soil on growth of Ocimum basilicum. Journal of El-Mestansrea, 22(4):91-99.

El-Naggar, A.H.M.; Hassan, M.R.A.; Shaban, E.H. and Mohamed, M.E.A. (2015). Effect of organic and biofertilizers on growth, oil yield and chemical composition of the essential oil of Ocimum basilicum, L. plants. Alex. J. Agric. Res., 60(1):1-15.
Helmy, T.A. (2016). Influence of Some Agricultural Treatments on Cumin Plant. Ph.D. Thesis, Fac. Agric. Minia Univ.

Jackson, M.L. (1973). Soil Chemical Analysis Englewood Cliffs., New Prentice-Hall INC., New York.

Khafi, M. (2003). Basil-Production and Processing Ferdowsi Univ. Mashhad Publication. Mashhad, Iran 195 pp.

Khalil, S.E.; Abd El-Aziz, N.G. and Abou Leil, B.H. (2010). Effect of water stress and ascorbic acid on some morphological and biochemical composition of Ocimum basilicum plant. J. American Sci., 6(12):33-44.

Massoud, Hekmat Y.A. (2006). Effect of phosphorus fertilization levels and foliar application with active dry yeast on growth, herb yield, essential oil productivity and chemical components of sage (Salvia officinalis, L.). J. Agric. Sci. Mansoura Univ., 31(10):6649-6665.

Mosaad, S.E. (2012). Physiological studies on sage plant. M. Sc. Thesis, Fac. Agric., Mansoura Univ., Egypt.

MSTAT-C (1986). A Microcomputer Program for the Design, Management and Analysis of Agronomic Research Experiments (Version 4.0), Michigan Stat Univ., U.S.

Nassar, M.A.; Mohamed, U.E. and Azoz, S.N. (2015). Influence of foliar spray with yeast extract on vegetative growth, yield of fresh herb, anatomical structure, composition of volatile oil and seed yield components of basil plant (Ocimum basilicum, L.). Int. J. of Advanced Research, 3(10):978-993.

Patel, K. and Kushwaha, N.K. (2013). Studies on influence of spices, nitrogen and spacing on parameters of plant growth at various stages of basil. Int. J. of Pharmacy \& Life Sci., 4(10):3028-3034.

Sadeghi, S.; Rahnavard, A. and Ashrafi, Z.Y. (2009). The effect of plant density and sowing date on yield of basil (Ocimum 


\section{M.A.H. Abdou et al.}

basilicum, L.) in Iran. J. of Agric. Shehata, A.M. (2013). Response of Guar Tecnol., 5(2):413-422. Plants to Some Agricultural Treatments.

Salman, A.S. (2006). Effect of biofertilization on Ocimum basilicum, L. Ph.D. Thesis, Fac. Agric., Minia Univ., plant. Egypt J. Agric. Res., 79(2):587Egypt.

606.

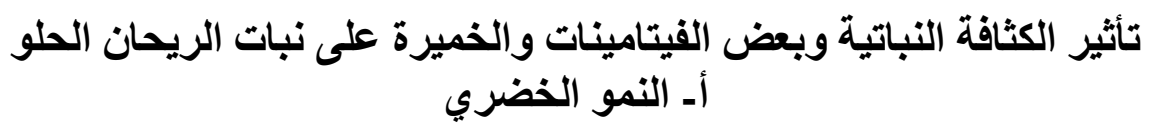

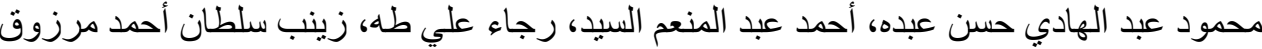

$$
\begin{aligned}
& \text { قسم البساتين، كلية الزراعة، جامعة المنيا، مصر. }
\end{aligned}
$$

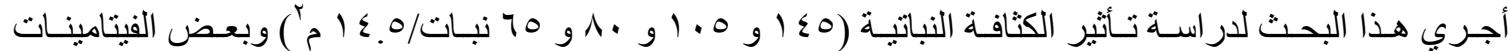

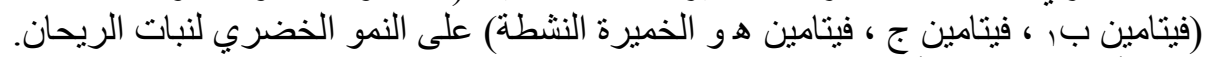

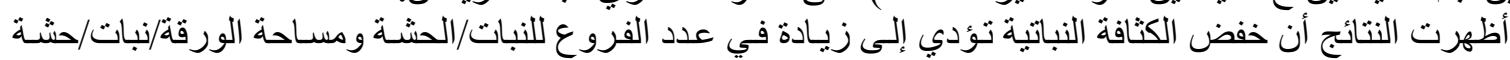

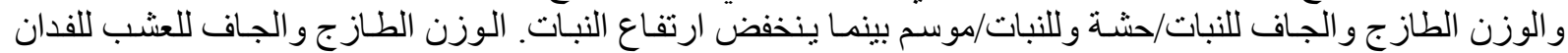

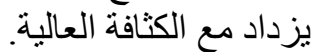

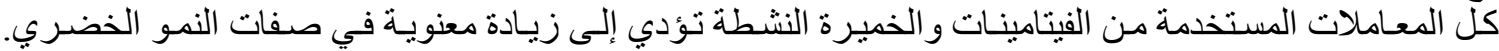

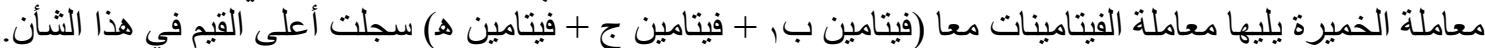

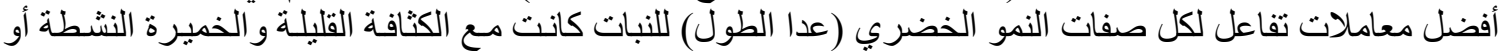

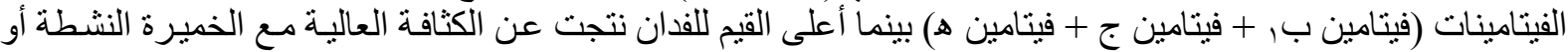
فيتامين ب, + فيتامين ج + فيتامين هـ 

